\title{
Circuit
}

Musiques contemporaines

\section{Le quatuor à cordes : un genre dépassé ?}

\section{Jean-Jacques Nattiez}

Volume 11, numéro 2, 2000

Le quatuor à cordes selon Schafer

URI : https://id.erudit.org/iderudit/004678ar

DOI : https://doi.org/10.7202/004678ar

Aller au sommaire du numéro

Éditeur(s)

Les Presses de l'Université de Montréal

ISSN

1183-1693 (imprimé)

1488-9692 (numérique)

Découvrir la revue

Citer cet article

Nattiez, J.-J. (2000). Le quatuor à cordes : un genre dépassé ? Circuit, 11(2),

73-76. https://doi.org/10.7202/004678ar d'utilisation que vous pouvez consulter en ligne.

https://apropos.erudit.org/fr/usagers/politique-dutilisation/ 


\title{
Le quatuor à cordes: un genre dépassé?
}

\author{
Jean-Jacques Nattiez
}

II est évident que, au XIX siècle, le genre "quatuor » a symbolisé le sommet de la musique pure et sublime, ce que Jean Portugais appelle, dans son texte d'introduction, "le canon de la musique sérieuse ". C'est probablement Nietzsche qui a été le premier, vers 1870, à en faire l'incarnation de la musique absolve: "Au moment des plus hautes révélations musicales, nous ressentons même involontairement la grossièreté de toute image [ou de tout affect] que l'on pourrait évoquer par souci d'analogie: les derniers quatuors de Beethoven par exemple font absolument honte à ce genre de comparaisons» (Nietzsche, 1973, p. 25). Autrement dit : le quatuor est encore plus élevé que ce qu'il y a de plus haut! Conception que partage Proust quelques décennies plus tard: par Vinteuil interposé, on sent dans les quatuors de Beethoven "une sorte de qualité morale et de supériorité intellectuelle», ils sont "la transposition, dans l'ordre sonore, de la profondeur» (Proust, 1988, p. 761).

Je me sens très proustien ici. D'où une précision, d'emblée, quant au contexte idéologique de mon propos. Portugais présente comme une valeur bourgeoise mais je sais qu'il se fait souvent l'avocat du diable dans son texte - l'idée que la musique pure serait plus "élevée» que la musique d'ambiance. Voilà qui me hérisse quelque peu. La qualité n'est pas bourgeoise. Ce n'est pas parce que la musique pop (je préférerais dire "la musique industrielle») nous a envahis et que ce sont les classes défavorisées et la jeunesse qui y trouvent leur satisfaction, que ces musiques sont, à l'inverse, progressistes ou démocratiques. Si on veut rentrer dans ces considérations, on pourrait aussi bien dire, à la manière de Adorno, que les catégories sociales qui fréquentent ces musiques sont des victimes des multinationales du disque. De plus, certains des compositeurs qui, après 1945, se sont confrontés au quatuor - au moins Nono, Xenakis et Boulez - se situaient à gauche, voire à l'extrême gauche de l'échiquier politique, ce qui semble prouver que la notion de "valeur bourgeoise " est historiquement très relative.

II n'en reste pas moins vrai, à mon sens, que se mesurer, même aujourd'hui, au genre quatuor, c'est accepter de relever ce que j'appellerai volontiers «le défi 
transcendantal beethovénien», ou de se situer volens nolens par rapport à lui. $C^{\prime}$ est, en tout cas, accepter de s'inscrire dans la lignée de la musique sérieuse, à moins qu'on ne décide de subvertir le genre, ce qui est en fait une manière de reconnaître combien le modèle du genre quatuor est puissant.

J'ai dit «qu'on le veuille ou non», car je soulignerai tout de suite que telle n'est pas nécessairement l'intention du compositeur. En s'appuyant sur une distinction qui m'est familière entre stratégies compositionnelles et stratégies de réception, il appert que l'entreprise, du point de vue historique, ne peut pas ne pas être interprétée ou évaluée a posteriori par rapport au paradigme du genre quatuor, même quand ce n'était pas l'objectif du compositeur ou quand il en avait modifié ou transformé la forme traditionnelle.

En raison du monument beethovénien et de la poursuite d'une tradition d'écriture du quatuor à cordes par des compositeurs majeurs du Xxe siècle, ovi, il y a un enjeu symbolique dans le choix même de ce genre. Murray Schafer me disait, au moment de l'événement qui lui a été consacré, qu'il avait beaucoup hésité à entreprendre son premier quatuor, précisément en raison de ce poids historique. Ce qui confirme, dans son cas, que l'exercice a été perçu comme une "épreuve initiatique», pour reprendre l'expression de Portugais. Et il n'est pas le premier. Chez Debussy et Ravel, les quatuors sont des œuvres de jeunesse, respectivement en 1894 et en 1902-1903; chez Boulez, le Livre pour quatuor est une œuvre de début de carrière (1948-1949), après les deux premières sonates pour piano, Le Soleil des eaux et Le Visage nuptial; et Kurtág fait de son quatuor (1959) l'opus 1 de son catalogue.

Il est également caractéristique qu'un certain nombre de compositeurs du XXe siècle aient entrepris la composition d'une série de quatuors. Debussy en projetait un second et avait d'emblée dénommé le sien "Premier quatuor». Webern en a composé trois, Schönberg et Carter quatre, Bartók six, et Murray Schafer sept. Même si ces compositeurs n'ont pas nécessairement pensé à Beethoven en les entreprenant, nous ne pouvons pas oublier que le maître de Bonn en a écrit dix-sept.

En se situant de facto, qu'il le veuille ou non, dans cette lignée, le compositeur peut souhaiter expérimenter ce qu'il peut écrire avec son langage spécifique ou nouveau, il peut souhaiter transformer le genre, ou les deux. Cela me paraît le cas de Boulez, qui a servi de point de départ aux questions de Portugais.

Il est très clair que tout ce que Boulez entreprend vise à favoriser son inscription dans l'histoire. Dans le cas du quatuor, il est passé d'une série de quatuors à la conception (avortée) d'un ensemble important et unique qui aurait dû occuper tout un concert (il y a le même objectif à la base des Structures pour deux pianos, de Pli selon pli, de Répons). En même temps, il désirait sans doute, avec son Livre, achever le genre, ce qui est un projet à la fois mallarméen et hégélien. Au plan de l'écriture, il désirait expérimenter ce que l'écriture postwebernienne permettait de réaliser pour cette formation. 
Ce fut un échec. La partition ne fut pas publiée intégralement et ce sont des extraits seulement qu'en a enregistrés le Quatuor Parennin. En 1968, il procédait à une première réorchestration de deux mouvements pour orchestre à cordes. En 1988, sans doute après avoir entendu, le visage contracté comme s'il écoutait la mauvaise œuvre de quelqu'un d'autre, la version primitive jouée à Villeneuvelès-Avignon par le Quatuor Arditti, il entreprenait une nouvelle version pour orchestre à cordes, qui fut présentée et enregistrée à Salzburg en 1992 lelle est accessible en vidéo-disque).

Mais une pièce pour orchestre à cordes, est-ce encore un quatuor? N'est-ce pas là un moyen de contourner l'ascèse, l'aridité et l'austérité des origines, non seulement dans le Livre pour quatuor version 1949 mais dans la Grosse Fuge de Beethoven, la seule pièce classique, soit dit au passage, que joue le Quatuor Arditti dans certains de ses concerts et qui en fait ainsi le symbole de la naissance de la modernité?

\section{Et Murray Schafer?}

Est-ce que ses Quatrième et Septième Quatuors, dans lesquels il ajoute une voix; est-ce que son Troisième Quatuor, dans lequel il introduit des cris de karaté ; est-ce que les Troisième et Quatrième Quatuors, dans lesquels il programme des déplacements du premier violon; est-ce que le Sixième Quatuor, qui est accompagné d'une performance de Taï Chi; le Septième Quatuor, qui suppose une mise en scène élaborée; sont encore des quatuors à cordes au sens strict du terme? N'est-on pas passé, avec ces quatre derniers exemples, à quelque chose qui se rapproche davantage du théâtre musical au sens de Kagel?

Dans la mesure où il y a très souvent un lien étroit, dans l'histoire de la musique, entre la formation instrumentale et le type d'écriture utilisé (des classiques à Bartók pour simplifier), il ne faut pas exclure la possibilité que le genre quatuor soit dépassé. La "perversion» du quatuor classique dans certains des quatuors de Murray Schafer prouverait que c'est bien le cas.

Mais je crois qu'il y a là, du point de vue historique et esthétique, quelque chose de plus. Dans la conférence présentée à la Chapelle historique du Bon Pasteur, notre compositeur faisait remarquer que, pour lui, Beethoven avait exploité le potentiel dialectique du quatuor, qu'il voyait dans ces œuvres une sorte de débat entre des ennemis intimes et que tout compositeur de quatuors était tiraillé entre la plénitude et le déchirement.

Cet aspect "dialogue» est explicitement présent chez Beethoven avec l'épigraphe du dernier mouvement du dernier quatuor, "La résolution difficilement prise. Le fautil ? II le faut», jeu de questions et réponses dont les instruments ont la charge d'imiter l'intonation alternée tout au long du mouvement. II y a donc déjà chez Beethoven un élément extramusical, même dans ce qui est censé incarner la musique absolve, à ceci près que l'épigraphe n'est ni lue ni entendue au cours de l'exécution, mais inscrite en tête de la partition. 
Parce qu'il peut être l'évocation d'une conversation, le quatuor appelle la voix. Schönberg l'introduit dans son Deuxième Quatuor. Murray Schafer le fait aussi. II ajoute des cris dans son quatuor de 1980, des gestes dans ceux de 1980 et de 1988. Je fais exprès de souligner les dates. La musique pure beethovénienne repoussait le parlé et le chanté dans les marges du quatuor. Murray Schafer les intègre. Ce faisant, il assume l'impureté musicale typique du postmodernisme actuel. Le genre quatuor est-il caduc ou dépassé pour autant?

Une seule question compte, quels que soient les moyens utilisés, quel que soit le genre par rapport auquel le compositeur se situe explicitement ou implicitement: le résultat, du point de vue de la finesse de l'écriture et de la force expressive, est-il esthétiquement réussi ? Grâce à l'entreprise heureuse du Quatuor Molinari, il nous fut possible de répondre positivement à cette interrogation lors de l'événement Murray Schafer. Avec lui, le genre quatuor n'a certes plus la pureté de ses origines historiques, mais le voilà transfiguré, transcendé. Une re-naissance sous une forme autre, sans que le lien avec le quatuor classique soit pour autant tranché.

Et pour ce long moment de bonheur et de qualité, que grâces soient rendues aux interprètes et au compositeur.

\section{Bibliographie}

NIETZSCHE, F. (1973), «Über Musik und Wort», Sprache, Dichtung, Musik, Tübingen, J. Knaus éd.

PROUST, M. (1988), La Prisonnière, À la recherche du temps perdu, nouvelle édition par J. Y. Tadié, Paris, Gallimard, Bibliothèque de la Pléiade, tome III. 